\title{
Influence of the substrate lattice structure on the formation of quantum well states in thin In and $\mathrm{Pb}$ films on silicon
}

\author{
Dil, J H ; Hülsen, B ; Kampen, T U ; Kratzer, P ; Horn, K
}

\begin{abstract}
The substrate lattice structure may have a considerable influence on the formation of quantum well states in a metal overlayer material. Here we study three model systems using angle resolved photoemission and low energy electron diffraction: indium films on $\mathrm{Si}(111)$ and indium and lead on $\mathrm{Si}(100)$. Data are compared with theoretical predictions based on density functional theory. We find that the interaction between the substrate and the overlayer strongly influences the formation of quantum well states; indium layers only exhibit well defined quantum well states when the layer relaxes from an initial face-centred cubic to the bulk body-centred tetragonal lattice structure. For $\mathrm{Pb}$ layers on $\mathrm{Si}(100)$ a change in growth orientation inhibits the formation of quantum well states in films thicker than 2 ML.
\end{abstract}

DOI: https://doi.org/10.1088/0953-8984/22/13/135008

Posted at the Zurich Open Repository and Archive, University of Zurich

ZORA URL: https://doi.org/10.5167/uzh-45727

Journal Article

Accepted Version

Originally published at:

Dil, J H; Hülsen, B; Kampen, T U; Kratzer, P; Horn, K (2010). Influence of the substrate lattice structure on the formation of quantum well states in thin In and Pb films on silicon. Journal of Physics: Condensed Matter, 22(13):135008.

DOI: https://doi.org/10.1088/0953-8984/22/13/135008 


\title{
Influence of the substrate lattice structure on the formation of Quantum Well States in thin In and $\mathrm{Pb}$ films on silicon.
}

\author{
J H Dil ${ }^{1, *}$, B Hülsen ${ }^{1}$, T U Kampen ${ }^{1}$, P Kratzer ${ }^{2}$, and K Horn ${ }^{1}$ \\ ${ }^{1}$ Fritz-Haber-Institut der Max-Planck-Gesellschaft, Faradayweg 4-6, 14195 Berlin, \\ Germany \\ ${ }^{2}$ Fachbereich Physik, Universität Duisburg-Essen, 47048 Duisburg, Germany \\ *Present address: Physik-Institut, Universität Zürich, Winterthurerstrasse 190, 8057 \\ Zürich, Switzerland \\ E-mail: jan-hugo.dil@psi.ch
}

\begin{abstract}
The substrate lattice structure may have a considerable influence on the formation of quantum well states in a metal overlayer material. Here we study three model systems using angle resolved photoemission and low energy electron diffraction: indium films on $\mathrm{Si}(111)$ and indium and lead on $\mathrm{Si}(100)$. Data are compared with theoretical predictions based on density functional theory. We find that the interaction between the substrate and the overlayer strongly influences the formation of quantum well states; indium layers only exhibit well defined quantum well states when the layer relaxes from an initial face-centered cubic to the bulk body-centered tetragonal lattice structure. For $\mathrm{Pb}$ layers on $\mathrm{Si}(100)$ a change in growth orientation inhibits the formations of quantum well states in films thicker than $2 \mathrm{ML}$.
\end{abstract}

PACS numbers: 73.21.Fg, 68.55.Jk, 79.60.Dp

\section{Introduction}

Ultrathin metal films play an increasingly important role in many technologies and are the subject of intense scientific research. The formation of quantum well states (QWS) in such films when their thickness becomes comparable to the electron coherence length is nowadays well understood[1]. The electronic and structural properties of such systems have yielded many unexpected results, such as the formation of magic heights[2], oscillations in the surface reactivity[3], sign and magnitude of the Hall coefficient[4], the formation of extra sub-bands[5], and a Rashba-type spin-splitting of the QWS[6]. In many instances, research on quantum well states has contributed to a better understanding of the physics of low-dimensional structures. Furthermore the evolution of quantum well states can be used as a probe for the structure of thin metal films[7].

With smaller film thickness, the volume-to-surface ratio decreases and the influence of 
the interface between the metal overlayer and the substrate increases. In the present paper, the influence of the lattice structure of the substrate on the formation of quantum well states in the metal overlayer will be discussed. Indium films on $\mathrm{Si}(111)$ and $\mathrm{Si}(100)$ are chosen as an example of heteroepitaxy where even the crystal symmetry of the metal and the substrate is different. The formation of QWS in $\mathrm{Pb}$ on $\mathrm{Si}(111)$ has been discussed previously[8], and is used in this work as a reference. We extend these studies to $\mathrm{Pb}$ on $\mathrm{Si}(100)$ to examine the influence of an energetically unfavourable growth direction on the metal overlayer imposed by the substrate. For the systems dealt with here we find that the substrate lattice structure may have a profound effect on the formation of QWS, and that in some cases confinement is hindered by such effects.

\section{Experimental and computational set-up}

The experiments were carried out in a stainless steel ultrahigh vacuum chamber with a base pressure of $1 \times 10^{-10}$ mbar. The measurements were performed at the $10 \mathrm{~m}$ normal incidence monochromator on the U125/2 undulator at BESSY II. Data were acquired using a Phoibos 100 electron energy analyzer (Specs Gmbh) equipped with a CCD detector, thus allowing for the simultaneous detection of emission angle and electron energy. The line scans presented in this work were obtained by cutting through the images without further angular integration. Under the typical experimental conditions used here the energy and angular resolution are better than $80 \mathrm{meV}$ and $0.2^{\circ}$, respectively.

The Si samples were cleaned by repeated flash annealing, after which the cleanliness was checked by the observation of a sharp LEED pattern, and the presence of the contamination sensitive surface states. $\mathrm{Pb}$ was deposited from a water-cooled Knudsen cell on the sample held at $100 \mathrm{~K}$, and the measurements were performed at the same temperature. At this temperature, no well ordered In layers form on $\mathrm{Si}(111)$, therefore the experiments involving In were performed at a sample temperature of $50 \mathrm{~K}$ using liquid helium for cooling of our flow cryostat. The deposition rate was initially calibrated using a quartz microbalance and further refined based on the development of the quantum well states as a function of coverage.

Band structure calculations were performed within the generalized-gradient approximation[9] to density functional theory (DFT), using the highly accurate allelectron full-potential linearized augmented plane wave (FP-LAPW) method implemented in the Wien2k code[10].

\section{Results and discussion}

\subsection{Structural properties of In films on Si(100) and Si(111)}

The detailed structure of the metal-semiconductor interface, and of the first few layers of the metal film can be of substantial influence on the occurrence of quantum well states from the metal bands. A major difference exists between the growth mode on the two 


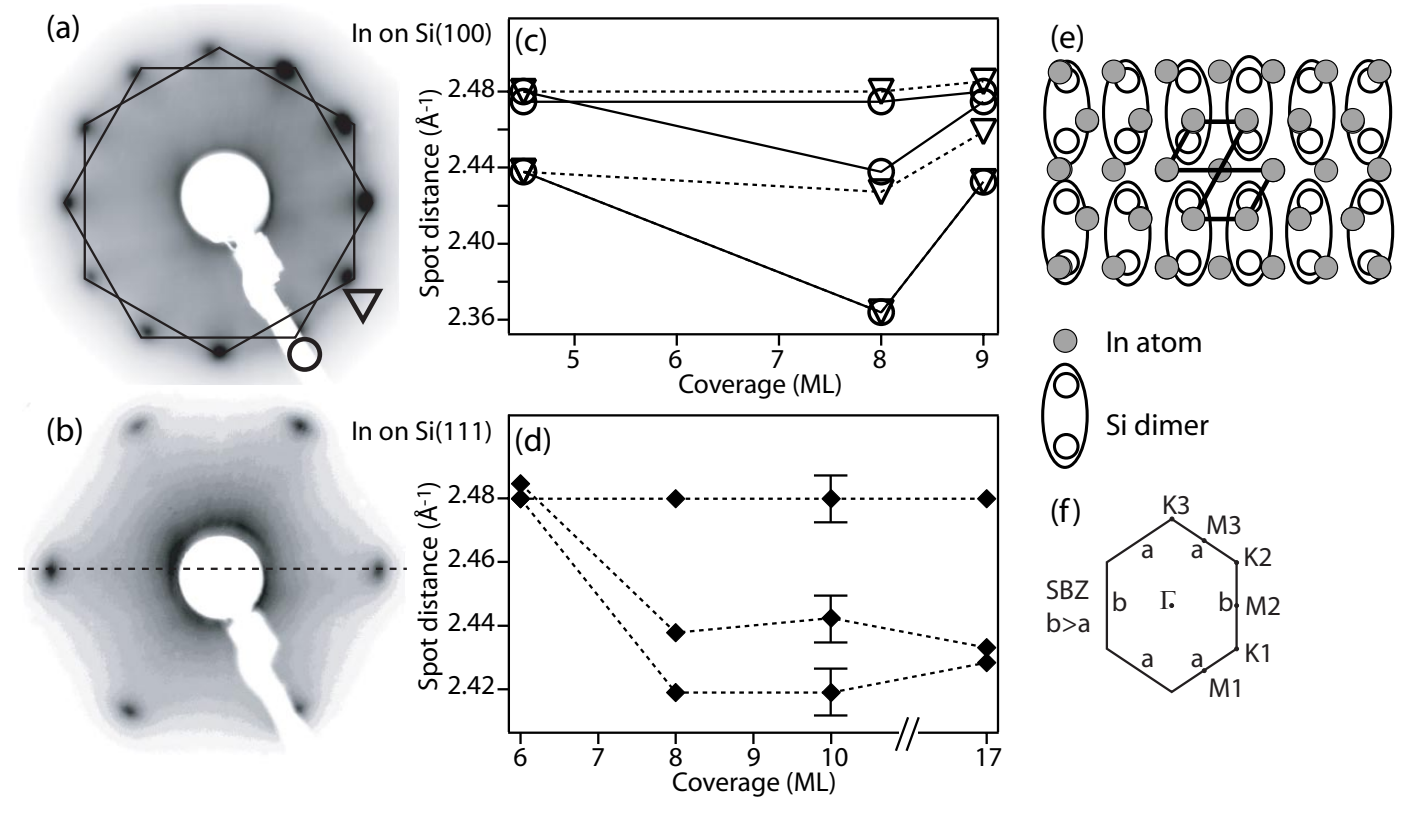

Figure 1. (a and b) LEED patterns for a $10 \mathrm{ML}$ thick layer of In grown on $\mathrm{Si}(100)$ (a) and on $\operatorname{Si}(111)$ (b). The dashed line in (b) indicates along which direction intensity traces are obtained. The hexagons and symbols in (a) refer to the symbols used in (c). (c and d) LEED spot distances as a function of In coverage on (c) $\operatorname{Si}(100)$ and (d) $\mathrm{Si}(111)$ demonstrating the presence of $b c t$ and $f c c$ In layers. The different symbols in (c) represent the different domains and the lines connect the geometrical orientations that belong together. The error bars for $10 \mathrm{ML}$ in (d) are representative for all the data points in (c) and (d). (e) Proposed lattice structure for one domain of In on $\mathrm{Si}(100)$. (f) (inset) bct surface Brillouin zone with $b>a$.

different silicon substrates used in this work as seen in Figure 1 where LEED patterns from a $10 \mathrm{ML}$ thick film of indium on $\operatorname{Si}(100)$ (a) and a film of identical thickness on $\mathrm{Si}(111)$ (b) are shown. The most striking distinction between the two patterns lies in the number of spots: 6 for the layer on $\mathrm{Si}(111)$ and 12 for the layer on $\mathrm{Si}(100)$. From the hexagonal LEED pattern we conclude that on both substrates the layers grow in the [111] direction. Since the $\mathrm{Si}(100)$ surface consists of two domains rotated $90^{\circ}$ with respect to each other, the indium overlayer also grows in two domains. This explains why there are 12 diffraction spots for $\operatorname{In} / \operatorname{Si}(100)$ and 6 for $\mathrm{In} / \mathrm{Si}(111)$. From the sharpness of the spots the good crystalline quality of the layer can be confirmed.

Bulk indium has a body-centred tetragonal crystal structure, which means that when cutting to obtain a [111] plane, the surface Brillouin zone has the shape of a slightly elongated hexagon as shown in Figure 1(f), where for clarity the distortion from the $f c c$ hexagon is exaggerated. In real space the distance $a$ is $3.8 \%$ larger than the interatomic distance $b$; in the SBZ this is inverted. This distortion of the perfect hexagon should also occur in LEED; the pattern should appear elongated in one direction. For the LEED images presented in Figure 1, this elongation is not directly obvious. In order to obtain a quantitative result, a numerical analysis of the LEED pattern was carried 
out using the following routine: along a horizontal axis, cutting exactly through two spots as indicated by the dashed line in Figure 1(b), an intensity trace is extracted from the image. The peaks in this spectrum are then fitted with a Gaussian function in order to deduce the exact peak positions. The use of spacings between two spots in the subsequent analysis, instead of their absolute position, reduces the influence of errors produced by a slight angular misalignment of the sample in front of the LEED instrument. The image is then rotated around the centre of the LEED pattern to obtain the next two spots along the horizontal axis, taking extra precaution not to distort the image in the process, and the analysis is repeated. Following this procedure, for $\mathrm{In} / \mathrm{Si}(111)$ three distances, and for $\mathrm{In} / \mathrm{Si}(100)$ six distances are obtained. Repeating this routine for several coverages results in a spot distance versus coverage plot. Figure 1(c) shows such a plot for In/Si(100), wherein the different symbols show the two domains as indicated by the hexagons and symbols in Figure 1(a). The results for In/Si(111) are shown in Figure 1(d); in both (c) and (d), lines connect those geometrical orientations (i.e. $\left.\bar{\Gamma}-\bar{K}_{1}, \bar{K}_{2}, \bar{K}_{3}\right)$ that belong together.

From the fact that for the indium films on $\mathrm{Si}(111)$, the spot distances for a coverage of 6 ML are the same in all three directions, we conclude that the In layer initially grows in a face-centred cubic structure. Since this structure for indium is only known in a highpressure phase[11], this is an example of pseudomorphic growth. For higher coverages, the layer relaxes towards a body-centred tetragonal crystal structure, indicated by the observation that two distances in reciprocal space are shorter than the other. For the 17 ML thick layer, the difference between the long and short axis is approximately $2 \%$. Since for bulk In this value is $3 \%$, the layers are not expected to be fully relaxed towards the bulk lattice at this film thickness. The observation of a phase transition from $f c c$ to $b c t$ is in line with STM and diffraction results for In/Si(111)-Pb- $(\sqrt{3} \times \sqrt{3})[12]$, where it has to be noted that our experiments have been performed at lower temperatures to enable the growth on the $(7 \times 7)$ reconstructed surface and that we do not observe a transition to (110) bct but the growth stays in the (111) direction.

From the general trend in the spacing of the LEED spots as a function of coverage it is clear that the growth mode of indium on $\mathrm{Si}(100)$ differs from that on $\mathrm{Si}(111)$. First, on $\mathrm{Si}(100)$ not all spot distances are identical for thinner layers, suggesting that the initial growth is bct and not $f c c$-like. The asymmetry in spot distances remains similar for higher coverages, indicating that the thinner layers are relaxed in a manner comparable to the thicker ones. For $\operatorname{In} / \mathrm{Si}(111)$ it was seen in the LEED pattern that in the bct growth region, one distance is larger than the two others; this would also be expected from the bulk lattice structure. Measurements for $\mathrm{In} / \mathrm{Si}(100)$ yield a different result; as explained below the asymmetry for the two different domains is opposite to each other. The triangular markers for domain 1 show one distance being larger than the others. On the other hand, domain 2, represented by the circles, shows one spot spacing being shorter than the other two. Superposition of the signal from these two domains rotated by $90^{\circ}$ with respect to each other results in the oval shape of the whole LEED pattern in Figure 1(a). The variations in distance and the general oval shape of the LEED 
pattern are also confirmed by the fact that, in order to rotate the image to horizontally align the next spots, variations in the rotation angle of as much as $3^{\circ}$ are needed. For a $180^{\circ}$ rotation, these variations cancel each other, i.e. rotating by exactly $180^{\circ}$ maps the pattern onto itself. From a comparison to LEED images obtained for the clean $\mathrm{Si}(100)$ substrate directly before indium deposition, it can be concluded that the odd distance (i.e. the distance shorter/longer than the other two) is aligned parallel to the dimer rows of the substrate. This alignment, perpendicular to the dimers, is then the only way in which the square symmetry of the substrate can be partly matched by the bct symmetry of the overlayer as schematically indicated in Figure 1(e). The asymmetry between the growth on the different substrate domains is most likely induced by the $\mathrm{c}(4 \times 2)$ low temperature reconstruction of $\mathrm{Si}(100)[13]$.

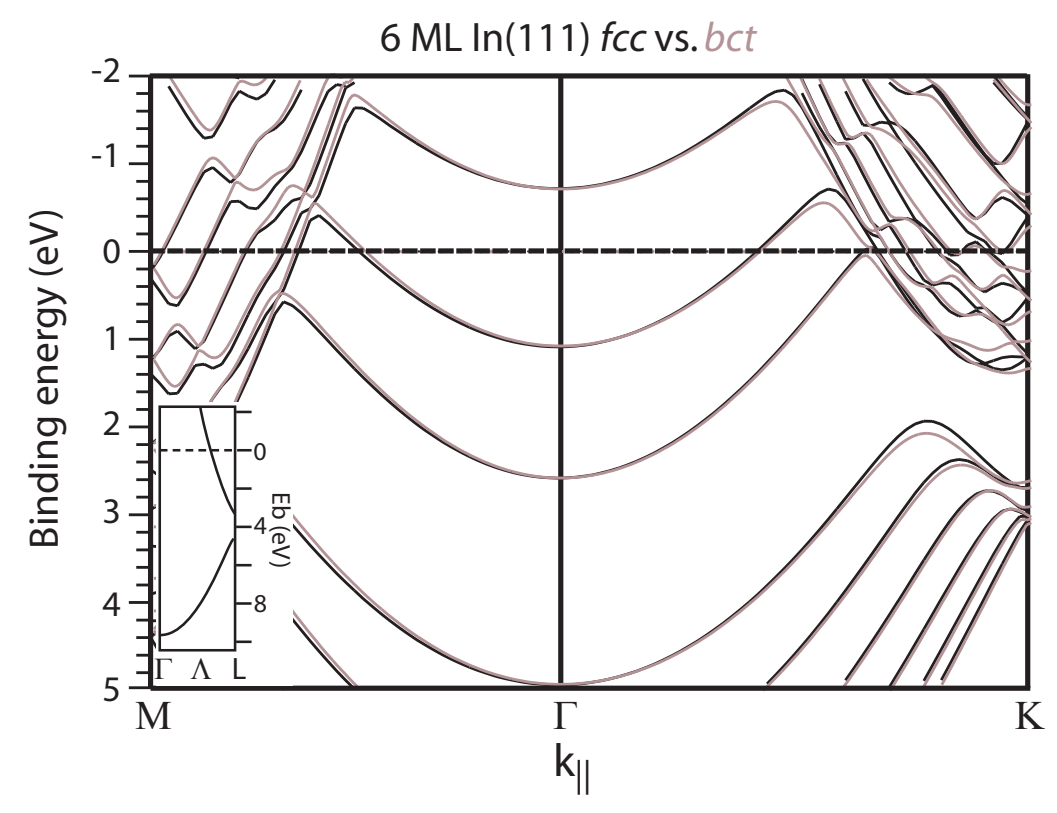

Figure 2. Comparison between the calculated band structure of a 6 ML thick freestanding (111) film of $f c c$ (black line) and bct (grey line) indium. The inset shows the In bulk band structure along the $\Gamma-\mathrm{L}$ direction

\subsection{ARPES of In films on Si(100)}

The relatively small lattice distortion away from the higher symmetry $f c c$ structure has only a limited effect on the predicted quantum well states, as can be observed in Figure 2, where DFT band structure calculations for $f c c$ and bct free-standing 6 monolayer (ML) thick indium slabs are displayed. The conduction band of indium has mainly $5 p$ character. The set of upward-dispersing parabolae around the zone centre represent the states originating from the confinement of the $5 p_{z}$ derived band in the quantum well, while the large number of states dispersing down towards the edge of the SBZ are 
due to quantization of the $5 p_{x, y}$ derived bands. As is directly visible from the figure the difference in the QWS energies between the two structures at the zone centre is negligible; small (tens of meV) differences only occur towards the edge of the Brillouin zone. These differences are too small to provide a distinction between either structure through ARPES measurements in view of the width of the bands as shown below. The calculated energies for the quantum well states at the parallel wave vector $k_{\|}=0$ in the topmost, partly occupied bulk In valence band[14] are displayed as empty circles in Figure 3(a): at a thickness of $3 \mathrm{ML}$ only one QWS at a binding energy of $2.0 \mathrm{eV}$ is occupied, at $6 \mathrm{ML}$ there are two, and at $9 \mathrm{ML}$ a third QWS is occupied. This is related to the shape of this band and its Fermi level crossing at about $\frac{1}{3}$ along the $\Gamma-\mathrm{L}$ line in the 3D band structure as shown in the inset of Figure 2[15].

Quantum well states for a single thickness as indicated in the plot are numbered start-
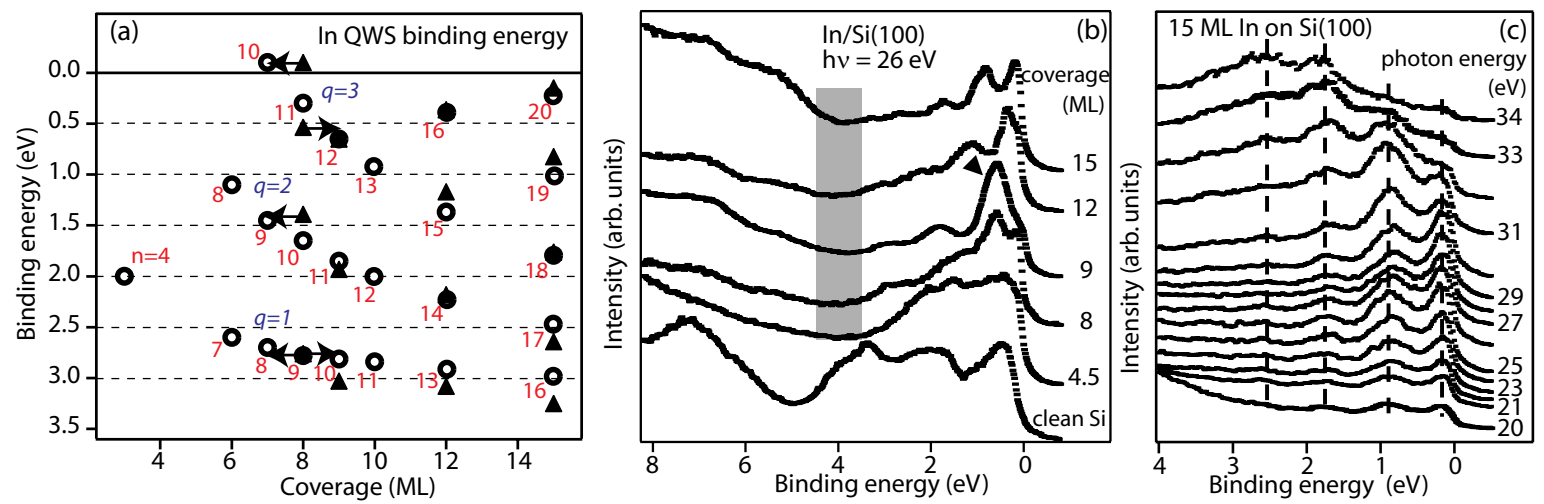

Figure 3. (a) Comparison between the measured QWS binding energies for $\operatorname{In} / \mathrm{Si}(100)$ (triangles) and the calculated QWS binding energies of freestanding In layers (open circles) as a function of thickness. The quantum number $n$ and the reduced quantum number $q$ are indicated. The arrows represent the observation that for a nominal coverage of $8 \mathrm{ML}$, the layer consists of a combination of 7 and $9 \mathrm{ML}$ thick regions. (b) EDCs of different coverages of $\mathrm{In}$ on $\mathrm{Si}(100)$ obtained at normal emission at a photon energy of $26 \mathrm{eV}$, the shaded area represents the fundamental bandgap in bulk In. (c) EDCs for $15 \mathrm{ML}$ of In on $\mathrm{Si}(100)$ obtained at normal emission as a function of photon energy. The dashed lines are added as a guide to the eye to indicate the absence of dispersion in the $\mathrm{k}_{\perp}$ direction.

ing from the deepest state located in the fully occupied valence band which has a binding energy of approximately $10 \mathrm{eV}$. The QWS in indium layers of different thickness that have a binding energy close to each other only differ in their quantum number by one; the quantum number $n$ of the state at $1.1 \mathrm{eV}$ in the $6 \mathrm{ML}$ film is 8 , for the state at 1.45 $\mathrm{eV}$ in the $7 \mathrm{ML}$ film $n=9$, and for the state at $0.3 \mathrm{eV}$ in the $8 \mathrm{ML}$ film $n=11$. Based on this a reduced quantum number $q$ can be defined for the In QWS as $q=n-N$, where $N$ is the layer thickness, resulting in branches of constant $q$ that move towards higher binding energies; they are also given in Figure 3(a).

The calculated quantum well state energies are compared with the experimental data 
derived by evaluating the binding energies in energy distribution curves as shown in Figure 3(b) for normal emission photoemission from clean $\mathrm{Si}(100)$ and several depositions of In on $\mathrm{Si}(100)$, obtained at a photon energy of $26 \mathrm{eV}$. For coverages of $8 \mathrm{ML}$ and more, several features are present that are not observed for the clean $\operatorname{Si}(100)$ surface. These lines show a strong dependence on the amount of indium deposited, which directly suggests that the features are derived from QWS. For the 4.5 ML thick film it is hard to distinguish any clear QWS and to determine their energies, although such features appear to be emerging. This is most likely caused by the fact that, at this coverage, there is no homogeneous layer, but rather islands of various heights, resulting in many states very close to each other. The shape of the photoemission background upon which the QWS features are placed at this coverage does, however, strongly resemble the one observed for higher coverages.

At these higher coverages sharp features, identified as QWS, are observed down to around $3.5 \mathrm{eV}$ below the Fermi level. Between 3.5 and $4.5 \mathrm{eV}$ there is a gap in the spectra, which corresponds to the bandgap in the $\Lambda$-direction for bulk In[14]. Below this gap, some broad lines can be observed. These are expected to originate from QWS in the lower valence band (see bulk band structure in the inset of Figure 2). In contrast to the upper valence band, for the lower valence band a new QWS is occupied for each additional layer because the band stretches across the entire Brillouin zone, therefore the states are close together. From this data it is clear that QWS are also formed in the lower valence band of indium; however, since these states, being much further away from the Fermi level, show strong lifetime broadening and are not well resolved due to an overlapping of states, they will not be discussed any further here.

Some of the QWS peaks in Figure 3(b) show a slight shoulder on the higher binding energy side, as for example the peak closest to the Fermi level in the 9 ML thick film. This belongs to the $10 \mathrm{ML}$ thick film, as indicated by the triangle. This indicates that the coverage is slightly more than $9 \mathrm{ML}$, and therefore areas with $10 \mathrm{ML}$ coverage exist. Such features will be taken into account in the evaluation of the data in order to more accurately determine the energies of the main features in the spectra. The binding energies for the most prominent QWS in thin In layers on $\mathrm{Si}(100)$ are indicated in Figure 3(a) as a function of coverage by solid triangles, while the QWS energies obtained from DFT calculations for free-standing indium films are indicated as open circles.

For an $8 \mathrm{ML}$ indium film, it is clear that the experimental QWS energies do not match the calculated results. From a comparison with the binding energies obtained by DFT, the QWS measured for a $8 \mathrm{ML}$ thick film can be ascribed to a combination of a 7 and $9 \mathrm{ML}$ thick film. This means that the $8 \mathrm{ML}$ film is not formed at all!. A possible explanation for this is the influence of quantum size effects (QSE) on film growth, similar to those responsible for the formation of stable and unstable islands heights in other systems[16, 17, 18, 19]. The QWS expected for an $8 \mathrm{ML}$ thick film is very close to $E_{F}$ which significantly increases the total energy of the system. When the QWS observed after deposition of $8 \mathrm{ML}$ of In are properly assigned, as discussed above and indicated by the arrows, the measured energies match the calculated ones very well. We therefore 
conclude that indium on $\mathrm{Si}(100)$ grows in a layer-by-layer fashion for coverages of $9 \mathrm{ML}$ or more. It seems that below this coverage, a mixture of layer heights is formed, which corresponds to the data for both the 4.5 and $8 \mathrm{ML}$ depositions. This interpretation is analogous to the inverse Stranski-Krastanov growth observed for Ag on $\operatorname{GaAs}(110)[16]$ where, after initial island growth, from a certain coverage onward atomically smooth layers are formed.

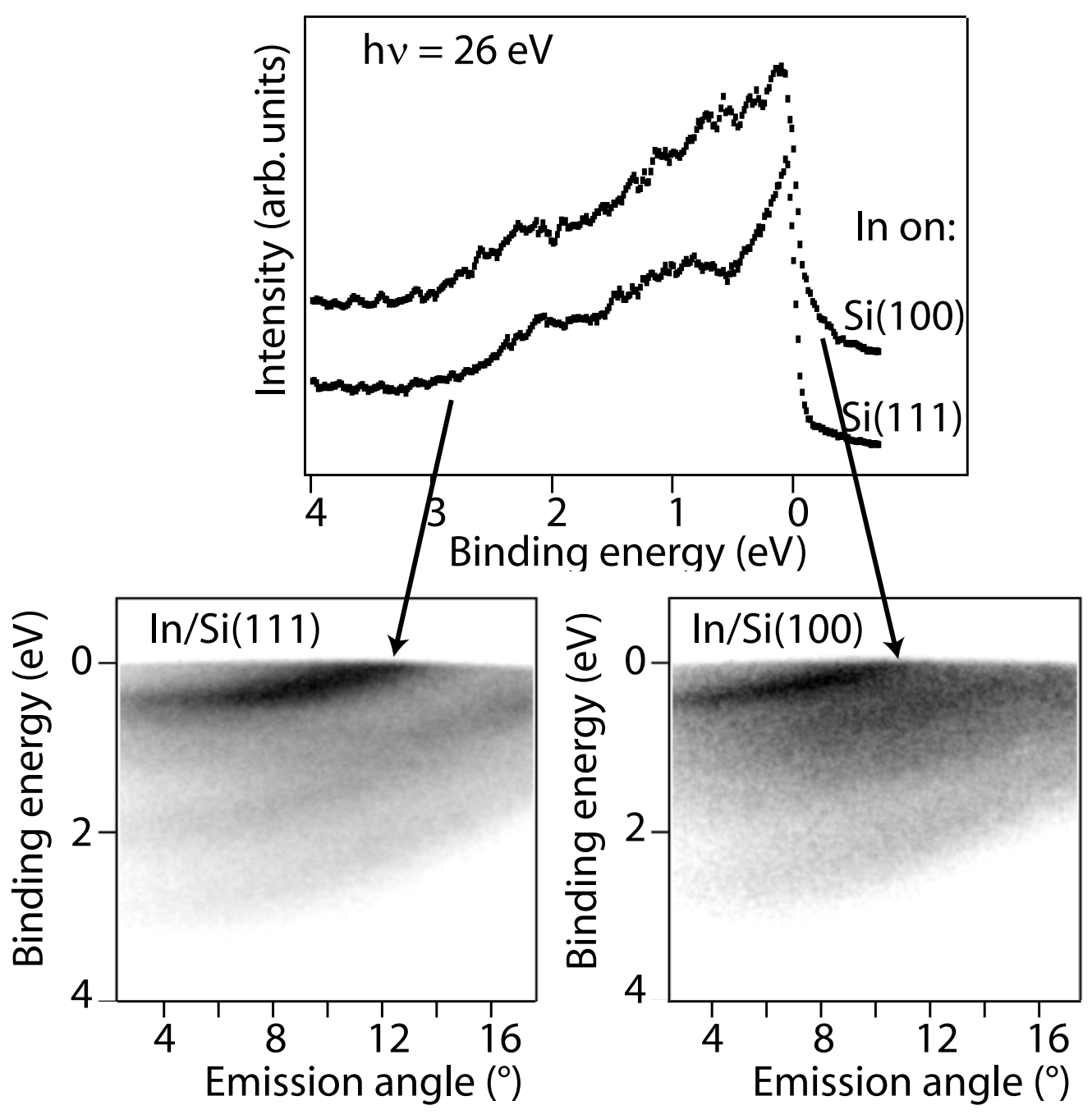

Figure 4. Off-normal emission images for $10 \mathrm{ML} \mathrm{In} / \mathrm{Si}(111)$ (left) and $\mathrm{In} / \mathrm{Si}(100)$ (right) at a photon energy of $26 \mathrm{eV}$. The arrows indicate where the spectra in the upper part have been extracted. Note that the colour scale of the images has been inverted to obtain a better contrast.

The occurrence of QWS is not influenced by the growth of two different domains of indium overlayers. In the center of the surface Brillouin zone, the $\Gamma-\mathrm{M}$ direction of domain 1 and the $\Gamma-\mathrm{K}$ direction of domain 2 exactly overlap (see Figure 1 ). The deviation between the energy bands in the two directions, which can only be probed 
simultaneously in photoemission, increases for larger $\mathrm{k}_{\|}$values. Figure 4 shows a comparison between an energy versus angle image obtained towards the edge of the SBZ in the $\Gamma-\mathrm{M}$ direction for $10 \mathrm{ML}$ of In on $\mathrm{Si}(111)$ (left), and on $\mathrm{Si}(100)$ (right). The details of the band structure will be discussed in the next section, however in the image obtained for In/Si(111), the features are significantly sharper than for $\mathrm{In} / \mathrm{Si}(100)$. This is confirmed by the energy cuts through the image (top), taken at the point where the topmost QWS crosses the Fermi level, as indicated by the arrows. For the In/Si(111) EDC, three individual peaks appear, whereas for In/Si(100) the peaks overlap and can hardly be resolved. Considering the fact that at the given thickness both layers are relaxed towards the bct lattice structure, the difference in spectrum quality is likely to be due to the mixture of domains. Because the different domains originate from the two domains of the substrate, suppressing the two-domain growth of indium on $\operatorname{Si}(100)$ would seem possible by using the single domain $\operatorname{Si}(100)$ substrate with a $4^{\circ}$ miscut.

Due to the confinement in the direction normal to the surface, QWS show no dispersion in this direction with the wavevector component $k_{\perp}$, and their binding energies should remain constant when changing the photon energy. Figure 3(c) shows the photon energy dependence of the QWS in a 15 ML thick indium film on $\mathrm{Si}(100)$, for photon energies between 20 and $34 \mathrm{eV}$. The individual spectra are obtained by taking a slice through an energy versus angle image at normal emission, and normalizing the intensities to the photon flux. Two clear observations can be made from this figure. First, the QWS show no noticeable dispersion as a function of photon energy, confirming the confinement of wave functions in the direction normal to the film surface. Secondly, there are strong variations in the intensity of the QWS as a function of photon energy. From a photon energy of $20 \mathrm{eV}$ onwards, the intensity of the QWS continues to increase up to a photon energy of around $29 \mathrm{eV}$. Above this photon energy, a behaviour is observable that shows strong similarities to results for QWS in well ordered Pb films[20]: the weight of the QWS intensities shifts away from the Fermi level, and follows the dispersion of the bulk band. This can be understood by considering the fact that the wave function in the quantum well consists of the rapidly oscillating Bloch wave function of the (bulk) metal convoluted with the quantum well state envelope wave function. Hence the QWS show some bulk-like character in their photoemission cross section, which is dominated here by the matrix element for transitions form the initial to the final state band for bulk indium. From this plot an optimum photon energy that combines a high cross section with a broad energy window in the analyser can be determined. This optimum is located around $26 \mathrm{eV}$, hence this is the photon energy that was used in most of our experiments involving thin indium layers.

\subsection{ARPES of In films on Si(111) $7 \times 7$}

For indium deposited on the reconstructed $\mathrm{Si}(111) 7 \times 7$ surface, the measured energy distribution curves are shown in Figure 5(a). The bottom spectrum is for clean $\operatorname{Si}(111)$, where two surface states can be observed; the Si-adatom-derived $S_{1}$ state at a binding 
energy of $0.2 \mathrm{eV}$, and the restatom-derived $S_{2}$ state at $\mathrm{E}_{\mathrm{B}}=0.8 \mathrm{eV}[21]$, as indicated in the plot. For a six layer thick film of indium, the Fermi edge is well developed, but only broad features are present in the valence band. The image from which this spectrum has been extracted (Figure 5(b)), shows the downward dispersing silicon valence band. Directly above this sharp feature, a broad band can be observed that shows almost no in-plane dispersion. This corresponds to the broad peak that occurs in the EDC for a 6 ML film in Figure 5(a). The fact that the silicon valence band is still clearly visible shows that, just as on $\operatorname{Si}(100)$, indium grows in islands for these low coverages. This is confirmed by the LEED image for this coverage(inset in Figure 5(b)). The inner spots, originating from the $\mathrm{Si}(111)$ surface, are more intense than the indium spots, which due to the difference in lattice spacing occur just outside the Si spots. Island-like growth for low coverages of In on $\mathrm{Si}(111)$ has also been observed in STM studies by Altfeder et al [22], which showed that the islands are slightly elongated in the direction along the step edges due to reduced diffusion perpendicular to the steps. The STM data further show that these islands are larger than $100 \mathrm{~nm}$ in all directions parallel to the surface.
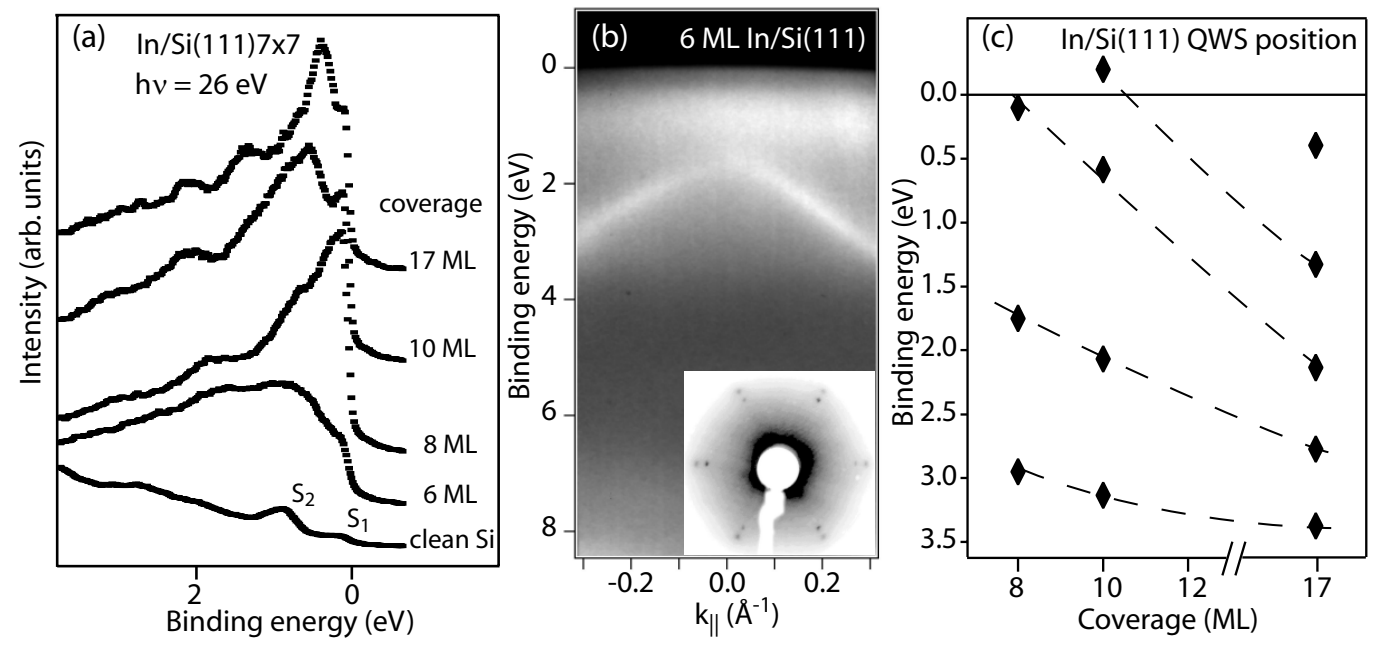

Figure 5. (a) EDCs for In deposited on $\operatorname{Si}(111) 7 \times 7$ at $50 \mathrm{~K}$, obtained at normal emission with a photon energy of $26 \mathrm{eV}$. (b) Energy vs. momentum image for $6 \mathrm{ML}$ In on $\operatorname{Si}(111) 7 \times 7$, (inset) corresponding LEED image. (c) QWS binding energy position as a function of coverage for In on $\operatorname{Si}(111) 7 \times 7$. The dashed lines correspond to QWS with the same quantum number $q$.

For coverages of $8 \mathrm{ML}$ or more, it appears that the islands close to form a smooth layer, because individual QWS can be clearly resolved. Moreover these states show a strong dependence on the total coverage. This agrees well with RHEED measurements of In growth on $\mathrm{Si}(111) 7 \times 7$ [23] and $\mathrm{Si}(111)(6 \times 6) \mathrm{Au}$ [24] substrates, where only after a coverage of $7 \mathrm{ML}$ clear layer-by-layer growth could be observed. The gap just below $4 \mathrm{eV}$ binding energy, indicated by the dark region in Figure 5(b) can be clearly seen, 
as in $\operatorname{In} / \mathrm{Si}(100)$. A major difference, however, is the intensity difference between the QWS within approximately $1 \mathrm{eV}$ below the Fermi level and the deeper lying states. For In/Si(100), the QWS intensity showed a gradual decrease away from EF, whereas here the transition is very abrupt. This cannot be a matrix element effect, because both data sets have been recorded at the same photon energy and because both films grow in the [111] direction. This discrepancy is therefore attributed to interface effects, as explained below.

Figure 5(c) shows the binding energy position of the primary QWS extracted from the EDC in Figure 5(a). It can be directly concluded that the appearance of QWS in indium layers grown on $\mathrm{Si}(111)$ follows the same rules as predicted by DFT and observed for $\mathrm{In} / \mathrm{Si}(100)$. For every three additional monolayers deposited, one additional QWS is

formed in the upper valence band because of the crossing of the Fermi level about $\frac{1}{3}$ along the distance from the Brillouin zone boundary. This periodicity of $3 \mathrm{ML}$ corresponds to the measured $3 \mathrm{ML}$ fluctuations in the conductance of In films on $\operatorname{Si}(111)(6 \times 6) \mathrm{Au}$ [24]. On the other hand STM measurements have suggested a 4 ML periodicity, in discrepancy with our observations [22].

Although the calculated data in Figure 2 suggest that the lattice structure should be of little influence on the electronic structure, in the In layers with an $f c c$ structure, no clear QWS are formed, and the energy versus momentum image in Figure 5(b) only shows a broad non-dispersing feature just above the silicon valence band maximum. We speculate that there is a connection between non-dispersive features in the quantum well spectra and the possibility to map the interface below the metal film by STM. For example, in a recent STM study[22] it was shown that the underlying $\mathrm{Si}(111) 7 \times 7$ surface could be imaged through indium islands with a height of as much as 7 ML. Because STM is a method in which electrons with $\mathrm{k}_{\|}$over a wide range may contribute, structural information buried below a metal layer cannot normally be obtained. This limitation is even valid for thin metal layers, where an electron can maintain its phase information over the distance travelled through the film. Because the electronic states observed for $f c c$ indium films are non-dispersing, they may provide a pathway through which an electron transported to the surface may retain local structural information from the interface. To understand this, we first note that the tip, when imaging, extracts or injects electrons with a sharply defined energy. If the same state has a constant energy over a broad $\mathrm{k}_{\|}$range, it is possible to describe the emitted electrons by a narrow wave packet in real space (broad in $\mathrm{k}$-space) which acts as a local probe for the transmission through the interface. This interpretation of the STM data is supported by the fact that for indium films thicker than $7 \mathrm{ML}$ (for which dispersing states develop) the In/ $\mathrm{Si}$ interface is no longer visible in STM. In the photoemission study presented here, this is confirmed by the formation of QWS with a free-electron-like dispersion. This means that electrons with a sharply defined energy must also have a narrow range of $\mathrm{k}$-vectors, and thus form a broad wave front in real space that averages over the local interface structure. The same physical principle may be responsible for the observation of the $\mathrm{Si}(111) 7 \mathrm{x} 7$ surface reconstruction through Pb layers of $100 \AA$ thickness[25], although in 
that system the lack of dispersion in the QWS sub-bands was suggested to originate from electron localization parallel to the surface which is still present for $\mathrm{Pb}$ layers of more than 20 ML thickness[26].

The limiting factor for the formation of QWS is not the $f c c$ lattice structure of the In films itself, since the DFT calculations indicate very little influence of the crystal structure (Fig. 1), nor is it likely to be the island-like growth because these islands are larger than $100 \mathrm{~nm}[22]$. In a recent theoretical approach on layer growth where the substrate was included in the analysis it was suggested that thin In layers are not allowed to form due to stress induced by the substrate[27]. The reason why QWS do not form could probably be elucidated by STM and X-ray diffraction studies. Based on the results presented here we can state that the transition to well ordered crystalline films that can accommodate QWS coincides with the transition from a $f c c$ to bct lattice structure, but that the mechanism responsible for this effect is not yet clear.

(a) $2 \mathrm{MLPb}$ on $\mathrm{Si}(100)$

(b) $15 \mathrm{ML} \mathrm{Pb}$ on $\mathrm{Si}(100)$

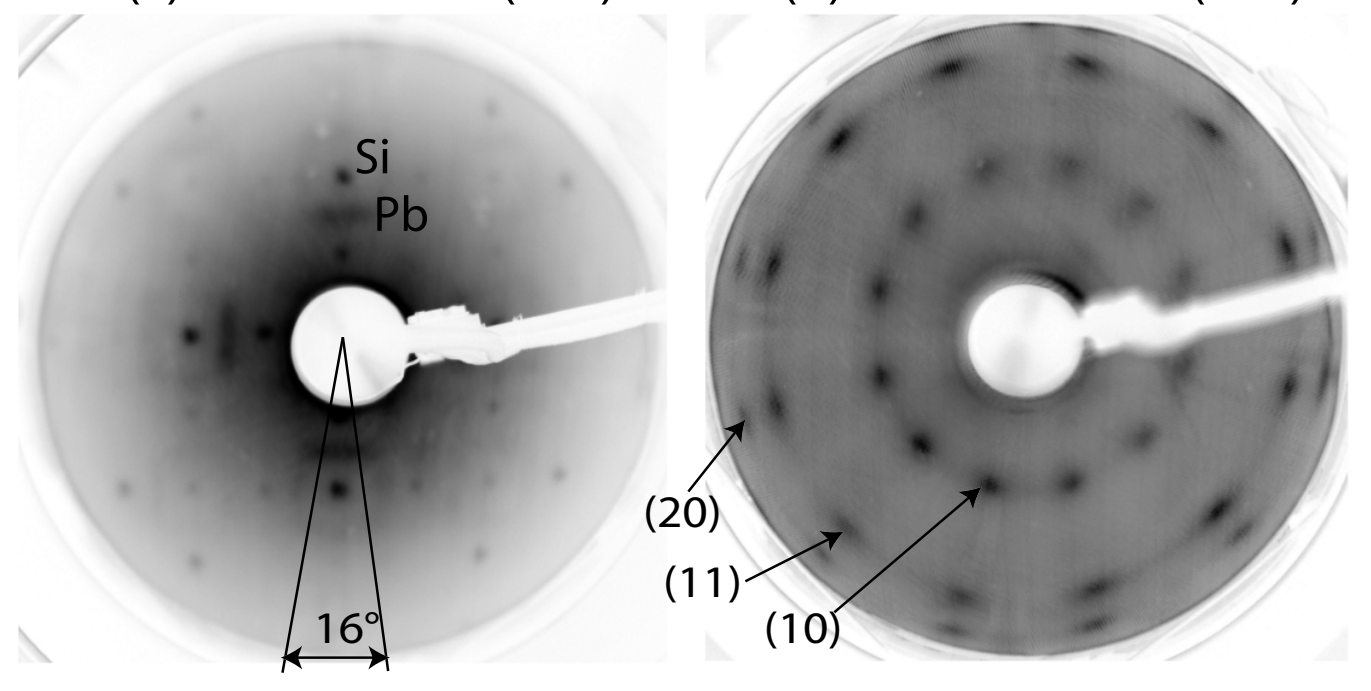

Figure 6. LEED patterns for (a) $2 \mathrm{ML} \mathrm{Pb}$ on $\mathrm{Si}(100)$ at $80 \mathrm{eV}$ and (b) $15 \mathrm{ML} \mathrm{Pb}$ on $\mathrm{Si}(100)$ at $105 \mathrm{eV}$.

\subsection{Structure of, and QWS in Pb films on Si(100)}

In this section we focus on a different system $(\mathrm{Pb}$ on $\mathrm{Si}(100))$ where the strain induced by the substrate also has a profound influence on the formation of QWS. Figure 6(a) shows the LEED pattern obtained after the deposition of $2 \mathrm{ML}$ of $\mathrm{Pb}$ on $\mathrm{Si}(100)$, at an electron energy of $80 \mathrm{eV}$. On the inside of the sharp spots from the square silicon substrate lattice, an elongated spot can be observed that is not present for clean $\mathrm{Si}(100)$. Upon closer inspection we find that this feature is actually composed of two separate 
spots, which can be assigned to a square lattice rotated by $8^{\circ}$ with respect to the $\mathrm{Si}(100)$ lattice, suggesting the formation of an epitaxial $\mathrm{Pb}(100)$ layer induced by the symmetry of the substrate. This layer should also leave its signature in ARPES, in that a QWS should show up at a binding energy of approximately $1.8 \mathrm{eV}$, in analogy with our derivation for indium above, and as predicted by DFT calculations[28]. Figure 7 shows a comparison between a $5^{\circ}$ off-normal emission photoemission image for clean $\mathrm{Si}(100)$ and the $2 \mathrm{ML}$ thick $\mathrm{Pb}(100)$ film formed on this substrate. The images were obtained with the dispersing angle along the horizontal direction of Figure 6(a). For the clean substrate, the heavy-hole (HH), light-hole (LH), and split-off (SO) bands[29] are identified and marked accordingly. After $\mathrm{Pb}$ deposition, an intense upward dispersing feature is formed, which does not shift with photon energy. Both this characteristic behaviour and the absence of this feature on the clean substrate suggests an assignment to either a quantum well state or an interface state. The good match to DFT results[28] indicates at the former. The binding energy of this state is slightly lower than predicted for a free-standing $\mathrm{Pb}(100)$ film of $2 \mathrm{ML}$ thickness, which may occur when the substrate influence is not negligible[20]. Emission from the silicon bands remains visible parallel to that from the $\mathrm{Pb}$ overlayer. The intensity distribution, however, is different from clean $\mathrm{Si}$, with higher intensities in regions close to the $\mathrm{Pb}$ QWS.

For higher coverages of $\mathrm{Pb}$ on $\mathrm{Si}(100)$, QWS are no longer observed; only a broad feature, dispersing with photon energy exactly like the $\mathrm{Pb}$ bulk band[30], is visible. This feature is comparable to observations for $\mathrm{Pb}$ on $\mathrm{Cu}(111)[20]$ and $\mathrm{Si}(111)[31]$ where the films have not been brought into thermal equilibrium, and is due to disorder at the metal/substrate interface. As explained above, the QWS wave function is composed of the rapidly oscillating Bloch wavefunction derived from the $\mathrm{Pb}$ atom spacing, modulated by the slowly varying QWS envelope function. This QWS envelope function only develops if a standing electron wave is formed, which in turn requires that the backscattering at the interface is coherent, and that the phase information is preserved while travelling through the layer. The LEED pattern of a $15 \mathrm{ML}$ film in Figure 6(b) provides a hint why these conditions are not met for Pb layers thicker than $2 \mathrm{ML}$ on $\mathrm{Si}(100)$ : From the third layer onwards, the $\mathrm{Pb}$ no longer grows in a square lattice, but in the hexagonal structure of a $\mathrm{Pb}(111)$ layer. Again the two domains of the $\mathrm{Si}(100)$ substrate are responsible for the growth of two domains in the $\mathrm{Pb}$ film, resulting in 12 spots being observed. This LEED pattern was recorded at an electron energy of $105 \mathrm{eV}$; at this energy the higher order diffraction spots are also visible (indicated in the image are the (10), (11), and (20) spots for one domain). Due to the $90^{\circ}$ rotation between the domains, the (11) and (20) spots from different domains almost overlap. Compared to $\mathrm{Pb} / \mathrm{Si}(111)(7 \times 7)[31]$, where QWS are formed, the spots are relatively sharp. Therefore the limiting factor is not the overall crystal structure of the overlayer. For coverages of approximately 4 ML, a combination of the square lattice from Figure 6(a) and the hexagonal lattice from Figure 6(b) is observed. This suggests that the $2 \mathrm{ML}$ thick $\mathrm{Pb}(100)$ layer survives underneath the $\mathrm{Pb}(111)$ film for higher coverages. This change in the orientation of the growth surface apparently inhibits coherent backscattering at the interface, and the 
formation of QWS, such that only the bulk-like band dispersion appears in the spectra. We explain the growth mode of $\mathrm{Pb}$ on $\mathrm{Si}(100)$ by a combination of two effects, namely
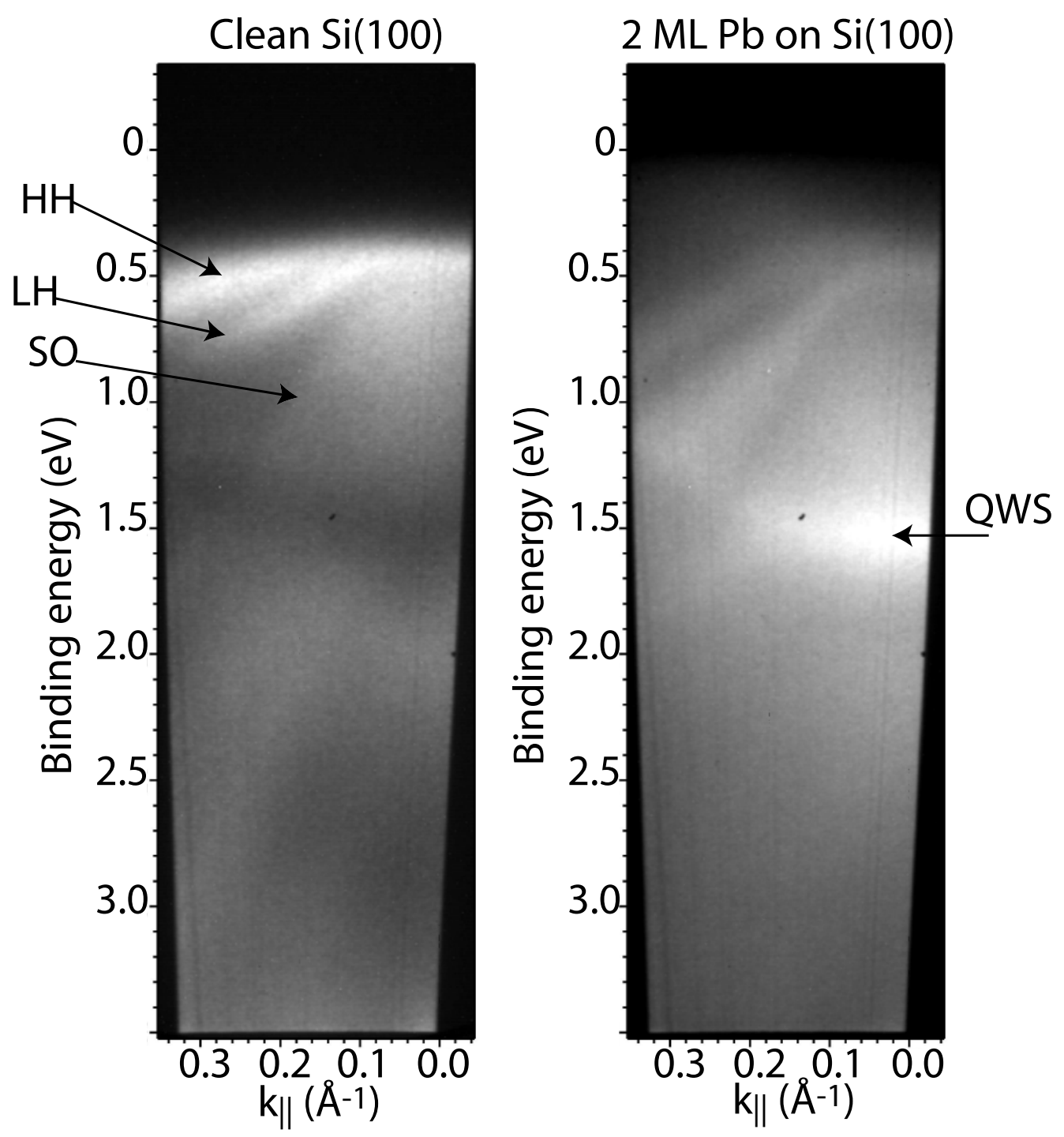

Figure 7. (left) Energy vs. momentum photoemission intensity image for clean $\mathrm{Si}(100)$ at a photon energy of $24 \mathrm{eV}$ with the heavy-hole $(\mathrm{HH})$, light-hole ( $\mathrm{LH})$ and split-off (SO) bands indicated. (right) Image obtained with the same parameters for 2 ML of $\mathrm{Pb}$ on $\mathrm{Si}(100)$, with the QWS indicated.

the orientation of the substrate, and electronic growth effects[32]. The initial growth being in the (100) direction is obviously due to the symmetry of the substrate; however, the formation of a $2 \mathrm{ML}$ thick layer and its survival under thicker films is rationalized by thermodynamic stability considerations. DFT calculations show that the surface energy per unit cell of a free-standing $2 \mathrm{ML}$ thick $\mathrm{Pb}(100)$ layer is about one third lower than the surface energy per unit cell of thicker $\mathrm{Pb}(100)$ films[28], and almost half of that for a 3 ML thick layer[33]. The formation of a $\mathrm{Pb}(111)$ film on a $\mathrm{Si}(100)$ substrate involves 
a considerable amount of strain in the overlayer, which increases the surface energy of the layer. Although the surface energy of $\mathrm{Pb}(111)$ layers[34] is much lower than that of $\mathrm{Pb}(100)$ layers over the full range studied, the extra energy due to strain shifts the energy minimum for $2 \mathrm{ML}$ thick films in the direction of the $\mathrm{Pb}(100)$ structure. Due to the large energy increase for the formation of a $3 \mathrm{ML}$ thick $\mathrm{Pb}(100)$ layer, the growth direction then suddenly changes to (111). This is in contrast to systems where the wetting layer is incorporated in the overlayer and therefore no extra barrier is formed. Surface X-ray diffraction experiments, especially in combination with ARPES, could give more insight in the details of this rather complicated growth.

\section{Conclusions}

We have characterized the influence of the substrate lattice structure on the formation of quantum well states, through a combination of LEED and photoemission studies and DFT calculations, comparing the systems In on $\mathrm{Si}(111)$ and $\mathrm{Si}(100)$, and $\mathrm{Pb}$ on $\mathrm{Si}(100)$. We find that in thin In films, well characterised quantum well states only form after the layer has relaxed into the bct lattice structure. Since our DFT calculations show that the In lattice structure has only a small influence on the electronic structure, the occurrence of the QWS may not simply be related to a transformation of the film structure, but may also depend on the interface structure. For $\mathrm{Pb}$ on $\mathrm{Si}(100)$ on the other hand, quantum size effects stabilize the $2 \mathrm{ML}$ thick $\mathrm{Pb}(100)$ film, but the tendency for energy minimization changes the growth surface for the thicker layers to the (111) direction. No QWS can form in films thicker than $2 \mathrm{ML}$ due to this change in growth direction.

For both systems a change in lattice structure influences the formation of quantum well states, where the change in growth direction for $\mathrm{Pb}$ has a more profound influence as the $f c c$ to bct relaxation in thin indium films.

\section{Acknowledgments}

This work was supported as part of the European Science Foundation EUROCORES programme SONS under programme MOL-VIC through funds from the Deutsche Forschungsgemeinschaft, and the EC Sixth Framework programme. J.H.D. acknowledges support by the International Max-Planck Research School Complex Surfaces in Materials Sciences. We gratefully acknowledge support by G. Reichardt and the BESSY staff.

\section{References}

[1] Chiang T -C 2000 Surf. Sci. Reports 39 181-235

[2] Budde K, Abram E, Yeh V and Tringides M C 2000 Phys. Rev. B 61 10602(R)

[3] Aballe L, Barinov A, Locatelli A, Heun S and Kiskinova M 2004 Phys. Rev. Lett. 93196103

[4] Jalochowski M, Hoffmann M and Bauer E 1996 Phys. Rev. Lett. 764227 
[5] Speer N J, Tang S J, Miller T, Chiang T C 2006 Science 314804

[6] Dil J H, Meier F, Lobo-Checa J, Patthey L, Bihlmayer G and Osterwalder J 2008 Phys. Rev. Lett. 101266802

[7] Luh D A, Cheng C M, Tsai C T, Tsuei K D and Tang J M 2008 Phys. Rev. Lett. 100027603

[8] Upton M H, Wei C M, Chou M Y, Miller T and Chiang T C 2004 Phys. Rev. Lett. 93026802

[9] Perdew J P, Burke K and Ernzerhof M 1996 Phys. Rev. Lett. 773865

[10] Schwarz K and Blaha P 2003 Comp. Mat. Sci. 28259

[11] Simak S I et al 2000 Phys. Rev. Lett. 85142

[12] Chen J, Hupalo M, Ji M, Wang C Z, Ho K M and Tringides M C 2008 Phys. Rev. B 77233302

[13] Wolkow R A 1992 Phys. Rev. Lett. 682636

[14] Ashcroft N W and Lawrence W E 1968 Phys. Rev. 175938 - 955

[15] Neuhold G 1996 Über die Valenzbandstruktur epitaktischer Metallfilme PhD Thesis, Freie Universität Berlin

[16] Smith A R et al 1996 Science $\mathbf{2 7 3} 266$

[17] Luh D A, Miller T, Paggel J J, Chou M Y and Chiang T C 2001 Science 2921131

[18] Otero R, Vazques de Parga A L and Miranda R 2002 Phys. Rev. B 66115401

[19] Dil J H, Kampen T U, Hlsen B, Seyller T and Horn K 2007 Phys. Rev. B 75 161401(R)

[20] Dil J H, Kim J W, Gokhale S, Tallarida M and Horn K 2004 Phys. Rev. B 70045405

[21] Uhrberg R I G et al 1985 Phys. Rev. B 31 3805; Hamers R J et al 1986 Phys. Rev. Lett. 561972

[22] Altfeder I B, Liang X, Yamada T, Chen D M and Narayanamurti V 2004 Phys. Rev. Lett. 92 226404

[23] Strozak M, Jalochowski M and Subotowicz M 1992 Acta Physica Polonica 81239

[24] Hnatyuk V and Strozak M 2007 J. Phys.: Condens. Matter 19396001

[25] Altfeder I B, Narayanamurti V and Chen D M 2002 Phys. Rev. Lett. 88206801

[26] Dil J H, Kim J W, Kampen Th, Horn K and Ettema A R H F 2006 Phys. Rev. B 73 161308(R)

[27] Prieto J E and Markov I 2007 Phys. Rev. Lett. 98176101

[28] Yu D, Scheffler M and Persson M 2006 Phys. Rev. B 74113401

[29] Hermann F 1958 RMP 30102

[30] Horn K, Reihl B, Zartner A, Eastman D E, Hermann K and Noffke J 1984 Phys. Rev. B 301711

[31] Mans A, Dil J H, Ettema A R H F and Weitering H H 2002 Phys. Rev. B 66195410

[32] Zhang Z, Niu Q and Shih C K 1998 Phys. Rev. Lett. 805381

[33] Wei C M and Chou M Y 2007 Phys. Rev. B 75195417

[34] Wei C M and Chou M Y 2002 Phys. Rev. B 66233408 